This item is the archived peer-reviewed author-version of:

\title{
Nonlinear optical properties of Ag nanoclusters and nanoparticles dispersed in a glass host
}

\section{Reference:}

Mai Hong Hanh, Kaydashev Vladimir E., Tikhomirov Victor K., Janssens Ewald, Shestakov Mikhail V., Meledina Maria, Turner Stuart, van Tendeloo Gustaaf, Moshchalkov Victor V., Lievens Peter.- Nonlinear optical properties of Ag nanoclusters and nanoparticles dispersed in a glass host

The journal of physical chemistry: C: nanomaterials and interfaces - ISSN 1932-7447 - 118:29(2014), p. 15995-16002 DOI: http://dx.doi.org/doi:10.1021/jp502294u

Handle: http://hdl.handle.net/10067/1186260151162165141 


\section{Nonlinear Optical Properties of Ag Nanoclusters and Nanoparticles Dispersed in a Glass Host ${ }^{\S}$}

Hong Hanh Mai, ${ }^{1 \ddagger}$ Vladimir E. Kaydashev, ${ }^{1 \ddagger}$ Victor K. Tikhomirov, ${ }^{2, \#}$ Ewald Janssens, ${ }^{1, *}$ Mikhail V. Shestakov, ${ }^{2}$ Maria Meledina, ${ }^{3}$ Stuart Turner, ${ }^{3}$ Gustaaf Van Tendeloo, ${ }^{3}$ Victor V. Moshchalkov, ${ }^{2}$ and Peter Lievens ${ }^{1}$

${ }^{1}$ Laboratory of Solid State Physics and Magnetism, KU Leuven, Celestijnenlaan 200D, 3001 Leuven, Belgium

${ }^{2}$ INPAC-Institute for Nanoscale Physics and Chemistry, KU Leuven, Celestijnenlaan 200D, 3001 Leuven, Belgium

${ }^{3}$ EMAT, Department of Physics, University of Antwerp, Groenenborgerlaan 171, 2020 Antwerp, Belgium

$\S$ This publication is dedicated to the memory of Victor K. Tikhomirov.

‡ These authors contributed equally to this work.

\# Deceased on 9 January 2014.

* ewald.janssens@fys.kuleuven.be 
ABSTRACT The nonlinear absorption of Ag atomic clusters and nanoparticles dispersed in a transparent oxyfluoride glass host has been studied. The as-prepared glass, containing 0.15 at.\% $\mathrm{Ag}$, shows an absorption band in the UV/violet attributed to the presence of amorphous $\mathrm{Ag}$ atomic nanoclusters with an average size of $1.2 \mathrm{~nm}$. Upon heat-treatment the Ag nanoclusters coalesce into larger nanoparticles that show a surface plasmon absorption band in the visible. Open aperture z-scan experiments using $480 \mathrm{~nm}$ nanosecond laser pulses demonstrated nonsaturated and saturated nonlinear absorption with large nonlinear absorption indices for the Ag nanoclusters and nanoparticles, respectively. These properties are promising, e.g., for applications in optical limiting and object's contrast enhancement.

KEYWORDS Ag clusters, nanoparticles, nonlinear absorption, oxyfluoride glass 


\section{Introduction}

Noble metal nanoparticles have attracted a lot of interest for application in optical devices due to their enhanced third order nonlinear optical response near the surface plasmon resonance (SPR). ${ }^{1}$ The optical Kerr effect, characterized by a complex third-order susceptibility $\chi^{3}$, which includes non-linear absorption and refraction coefficients, takes place in isotropic media where the second order optical nonlinear effect is forbidden. ${ }^{1,2}$ The third order nonlinear optical response of gold atomic clusters and nanoparticles dispersed in the liquid host of toluene, demonstrates a low threshold for optical non-linearity and low power limiting associated with the absence of absorption saturation. ${ }^{3}$ Large two-photon absorption cross sections for monolayer protected gold clusters are observed, which suggests future applications of these particles as optical limiting materials. ${ }^{4}$ The nonlinear optical properties of silver nano-colloids have also been shown to switch to saturated absorption at high laser irradiances. ${ }^{5}$

A liquid host for optical nonlinear particles has obvious disadvantages, such as poor mechanical and chemical robustness and high attenuation due to light scattering. Therefore, we were looking for a more durable and stable host for optical nonlinear noble metal clusters and nanoparticles. A glass host for example intrinsically provides possibilities for high optical transparency and preparation in different shaped bulk forms, including planar waveguides and fibers. ${ }^{6,7}$ Bulk glasses doped with Ag nanoclusters or nanoparticles homogeneously dispersed across the whole doped bulk glasses can be prepared by conventional melt-quenching methods as described in refs. [6,7], whereas doping by ion exchange produced nanoclusters that are embedded only in a thin layer near the surface of the glass. ${ }^{8}$ Silver nanoparticles are of particular interest because they are known to have optical absorption transitions with high oscillator strengths. Quenching of the oscillator strength in small Au, Ag, and Cu clusters is usually 
associated with enhanced s-d hybridisation. For silver clusters the s-d hybridization is less pronounced than for gold clusters of the same size. ${ }^{9-11}$ Thus, higher nonlinear absorption coefficients are expected for Ag. In addition, maximal quantum efficiencies that are reported in literature for Ag nanoclusters (64-69\%) ${ }^{12,13}$ are larger than for $\mathrm{Au}(41 \%) .{ }^{14}$ The Ag nanoclusters in the glass host used in this work do emit a broad white luminescence with a quantum yield above $20 \%{ }^{6}$

In this work the nonlinear absorption of $\mathrm{Ag}$ clusters (average size of $1.2 \mathrm{~nm}$ ) and $\mathrm{Ag}$ nanoparticles (average sizes of $2.4 \mathrm{~nm}$ and $2.8 \mathrm{~nm}$ ) dispersed in a transparent oxyfluoride glass host is studied by z-scan experiments using $480 \mathrm{~nm}$ nanosecond laser light. Non-saturated and saturated nonlinear absorption was found for the Ag atomic clusters and Ag nanoparticles, respectively. An energy level diagram with the respective excitation transitions is proposed for the Ag nanoclusters and nanoparticles involved in the observed nonlinear phenomena.

\section{Experimental}

The silver doped oxyfluoride glasses were prepared by conventional melt-quenching methods using chemicals supplied by Alfa Aesar. Hereto $\mathrm{SiO}_{2}, \mathrm{Al}_{2} \mathrm{O}_{3}, \mathrm{CdF}_{2}, \mathrm{PbF}_{2}, \mathrm{ZnF}_{2}$, and $\mathrm{AgNO}_{3}$ powders were placed in a Pt crucible. The chemical formula of the glass host is 33( $\left(\mathrm{SiO}_{2}\right)$ 9.5 $\left(\mathrm{AlO}_{1.5}\right) 32.5\left(\mathrm{CdF}_{2}\right) 19.5\left(\mathrm{PbF}_{2}\right) 5.5\left(\mathrm{ZnF}_{2}\right)$ mol\%. This oxyfluoride glass was selected because it is known to be a good glass former, which in addition is well capable of dissolving luminescent dopants such as Ag nanoclusters ${ }^{6,7}$ or rare-earth ions. ${ }^{15}$ A pure oxide host does not dissolve Ag nanoclusters/particles within its bulk; the fluoride component is required to dissolve nanoparticles. Doping was obtained by addition of $\mathrm{AgNO}_{3}$ to the batch that was melted in a tube furnace at $1000{ }^{\circ} \mathrm{C}$ for 1 hour and afterwards cast into an $\mathrm{Al}$ mould at room temperature. Details 
of the preparation procedure of the bulk oxyfluoride glasses that are uniformly doped with Ag nanoclusters and nanoparticles have been reported elsewhere. ${ }^{6,7}$ Photographs of the samples are shown in the Fig. S1 of the Electronic Supporting Information - ESI. The as-prepared glasses doped with Ag nanoclusters have a light yellow color due to an absorption band in the UV/violet. Upon further heat-treatment in air at $350^{\circ} \mathrm{C}$ larger Ag nanoparticles are formed and an Ag-related plasmon absorption band appears in the visible part of the spectrum, giving the glasses a red color. Note that the heat treatment was carried out at $350{ }^{\circ} \mathrm{C}$, which is below the glass transition temperature of $370{ }^{\circ} \mathrm{C} .{ }^{15}$

Transmission Electron Microscopy (TEM) investigations were carried out using a Philips CM 30 electron microscope operated at $300 \mathrm{kV}$. To do so, the glass samples were crushed in an agate mortar with ethanol and a drop of the suspension was placed onto a holey carbon grid. To obtain the size distribution, the diameters of roughly 75 particles were measured for each sample, assuming a spherical morphology. ADF-STEM images and energy-dispersive X-ray analysis (EDX) maps were acquired on an aberration-corrected Titan "cube" microscope, operated at $120 \mathrm{kV}$ acceleration voltage and equipped with a four-quadrant "Super-X" EDX detector. The convergence semi-angle $\alpha$ used was $22 \mathrm{mrad}$, the acceptance inner semi-angle $\beta$ used was 33 mrad.

Absorbance spectra were recorded with a Bruker Vertex $80 \mathrm{~V}$ Fourier Transform spectrometer. The nonlinear optical absorption of the glass samples was investigated by open aperture z-scan experiments, ${ }^{16,17}$ in which the thin transparent samples were translated longitudinally in a tightly focused laser beam and the transmittance measured in the far field. The nonlinear refraction of the studied samples was found to be negligible and is therefore not discussed here. Indeed, refraction induced by scattering at nanoparticles is known to be small if the particles' diameters 
are smaller than $10 \mathrm{~nm} .{ }^{18}$ As light source for the z-scan experiment a $480 \mathrm{~nm}$ pulsed laser beam with a Gaussian beam profile was used. The light originates from an optical parametric oscillator (OPO) pumped by a Quanta-Ray Nd:YAG laser (8 ns pulse duration and $10 \mathrm{~Hz}$ repetition rate). The laser beam radius $\omega_{0}$ was calculated to be $18 \mu \mathrm{m}$. Note that the laser beam is partially cut by a diaphragm. Taking into account the entire optical scheme, the Rayleigh length is estimated to be $14 \mathrm{~mm}$. This is much larger than the sample thickness of $1.35 \mathrm{~mm}$, satisfying the basic criteria of a z-scan experiment. The sample irradiance along the $\mathrm{z}$-scan was varied in the $10^{10}-10^{12} \mathrm{~W} / \mathrm{m}^{2}$ range. The nonlinear absorption coefficient and the absorption saturation irradiance are evaluated from the fit of the z-scan curves by direct numerical solution of the differential equations for the sample transmittance. No optical degradation of the samples was observed, even not at the highest applied sample irradiance $1.5 \times 10^{12} \mathrm{~W} / \mathrm{m}^{2}$.

\section{Results}

\section{Bright-field TEM imaging}

Fig. 1 shows bright-field TEM images of Ag nanoparticles and nanoclusters in the as-prepared (sample A), 6 hours heat-treated (sample B), and 38 hours heat-treated (sample C) glasses. An EDX analysis performed on the same sample in a previous study indicated a 0.15 at.\% total concentration of Ag in the as-prepared glass sample, and consequently in the heat-treated glass samples too, even though the batch contained $5 \mathrm{wt} \%$ of $\mathrm{AgNO}_{3}{ }^{18}$ One could speculate that such dissolution of $\mathrm{Ag}$ (0.15 at.\%) may be close to optimum, while the remaining Ag may evaporate in the melting process. It should be noted that all three samples are unstable under electron beam illumination conditions, and the Ag nanoparticles have the tendency to agglomerate. Therefore, all microscopy was performed using the shortest possible electron beam exposure of the samples. 
High resolution TEM imaging demonstrates that none of the smallest Ag clusters in the material are crystalline. No lattice fringes are observed in any of the HRTEM images, meaning all the smallest clusters are amorphous in nature.

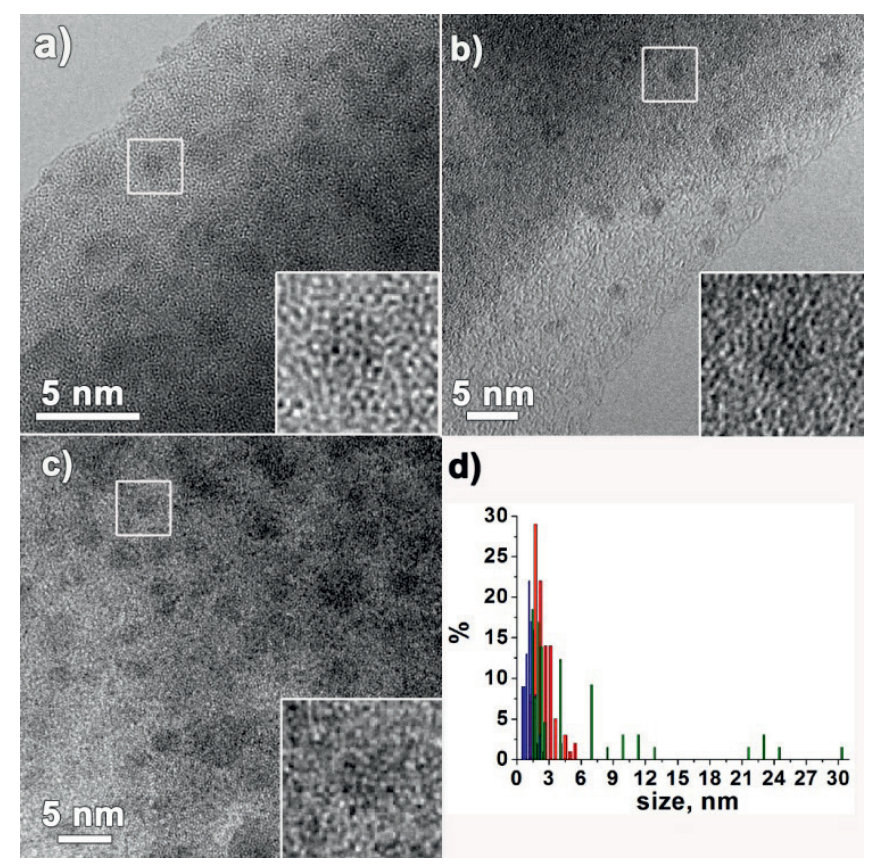

Figure 1. Bright-field TEM images of a) as-prepared (sample A), b) heat-treated for 6 hours (sample B), and c) heat-treated for 38 hours (sample C) Ag-doped glasses. The insets show enlarged images of the amorphous nanoclusters and nanoparticles present. d) Size distributions of the Ag clusters/nanoparticles present in the samples. Blue: asprepared, red: 6 hours treated, green: 38 hours treated.

A size distribution of the Ag clusters/nanoparticles is provided in Figure 1d. Individual size distributions of samples A, B, and C are available as Fig. S2 in the ESI. The average diameter (and median diameter) of the Ag nanoparticles in samples A, B, and C corresponds to $1.2 \mathrm{~nm}$ $(1.2 \mathrm{~nm}), 2.4 \mathrm{~nm}(2.3 \mathrm{~nm})$, and $2.8 \mathrm{~nm}(2.2 \mathrm{~nm})$, respectively. It is clear that upon increase of the sample treatment time, the average cluster/nanoparticle size also increases. The median sizes of sample B and C are very close to each other, as the longer treatment leads to the formation of only a small number of larger nanoparticles. The majority of particles in samples $\mathrm{C}$ have a diameter below $5 \mathrm{~nm}$. 


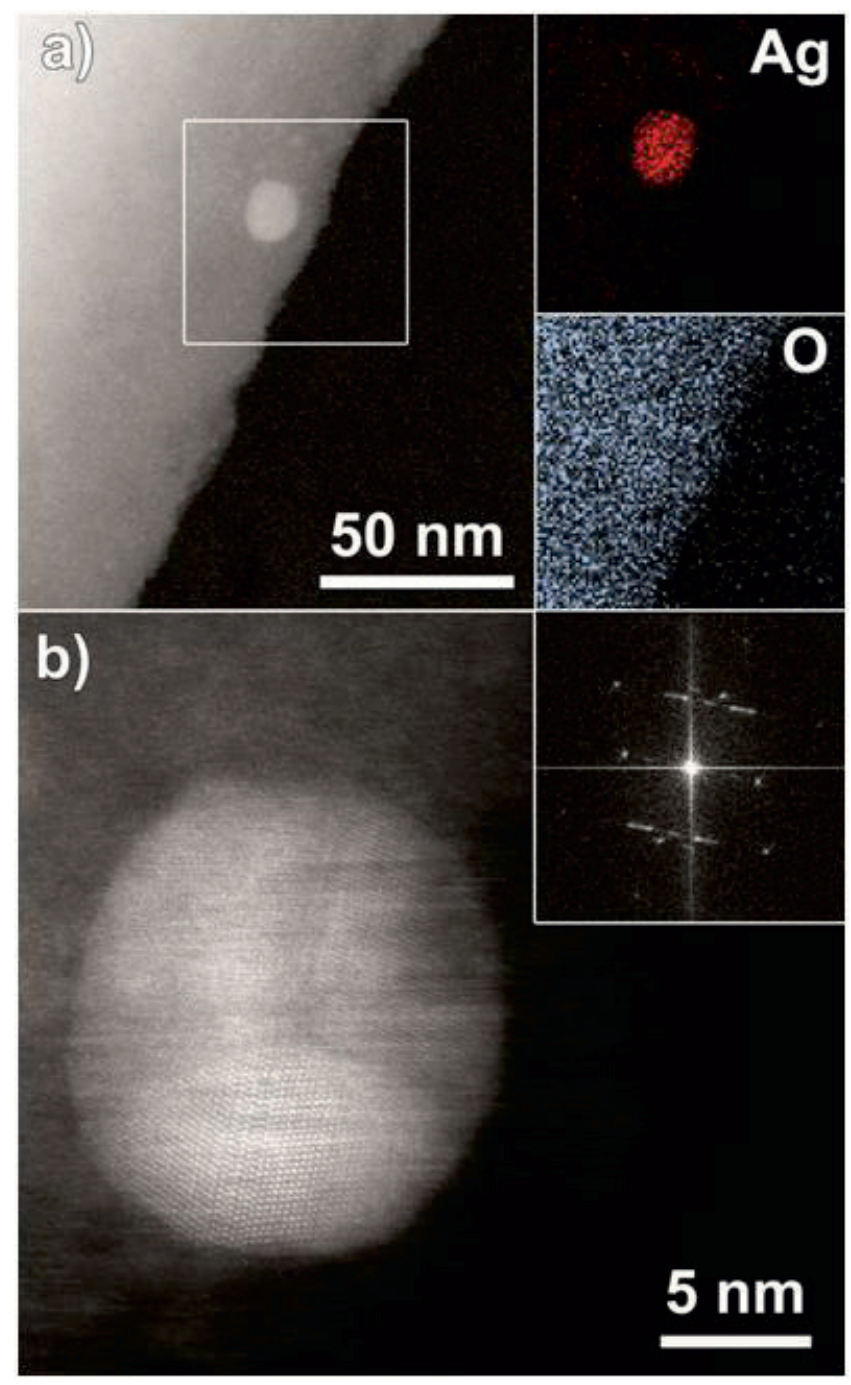

Figure 2. Composition of a large Ag nanoparticle in the 38 hours treated sample. a) ADF-STEM image of a single large Ag nanoparticle. The particle is composed of metallic Ag, as evidenced by the EDX maps for Ag and O. b) HR-ADF-STEM image of the same nanoparticle, with inset Fourier transform pattern, demonstrating that the particle is polycrystalline in nature.

In the case of the 38 hours sample (sample C), significantly larger nanoparticles, up to tens of nanometers in diameter, are also infrequently present in the glass matrix. In order to investigate the nature of these larger Ag nanoparticles, energy-dispersive X-ray analysis mapping and (highresolution) annular dark-field scanning transmission electron microscopy (ADF-STEM) was 
carried out (Figure 2). In figure 2a, an ADF-STEM image of a rare, large spherical Ag nanoparticle in the 38 hours treated sample is displayed. The EDX maps for Ag and O presented on the right clearly demonstrate that the nanoparticle is composed of metallic Ag, and not of, e.g., AgO. No other elements from the glass matrix are enriched in the nanoparticle, as proven by the EDX maps for Si, Al, Pb, Cd, F, and Zn (see Fig. S3 in the ESI). The high resolution ADFSTEM image of the same nanoparticle, presented in figure 2b, clearly shows that the nanoparticle is polycrystalline (also proven by the Fourier transform pattern in the inset). It must be stated that not all large Ag nanoparticles found in the sample are crystalline. An example of a large, amorphous Ag nanoparticle in the 38 hours treated sample is presented in the ESI (Fig. S4).

\section{Absorbance spectroscopy data}

The optical density (or absorbance) of the Ag doped glasses are shown in Fig. 3. The optical density is presented after correction for reflectance of $4 \%$ from each face of the sample. The absorbance spectrum of the undoped glass and sample A only show an onset of a pronounced absorption in the violet/UV part of the spectrum, corresponding to the absorption of the glass host and Ag nanoclusters consisting of several Ag atoms. ${ }^{3,6}$ A less intense absorption tail, stretching from 400 to $700 \mathrm{~nm}$, is observed in the visible range but no pronounced bands are observed. This observation is in agreement with previous computational work on small free standing Ag clusters, which predicted no intense absorption bands below $3 \mathrm{eV} \cdot{ }^{9,20,21}$ The spectrum of sample C shows an intense SPR band in the visible part of the spectrum (around 470 $\mathrm{nm}$ ) with a full width at half maximum of $150 \mathrm{~nm}$, which is typical for Ag nanoparticles with diameter of a few nanometers. ${ }^{22,23}$ The plasmon absorption band becomes more intense with increasing heat treatment time. These effects are related to an increase of size of the Ag 
nanoparticles with heat treatment. The linear absorption coefficients $\alpha_{0}$ at $480 \mathrm{~nm}$ (the wavelength used for the z-scan experiments) are derived from the optical density and range from $3.8 \times 10^{2} \mathrm{~m}^{-1}$ for sample A to $8.9 \times 10^{2} \mathrm{~m}^{-1}$ for sample C (see Table I). This increase of $\alpha_{0}$ with the particle size is in accordance with previous studies. $^{3}$

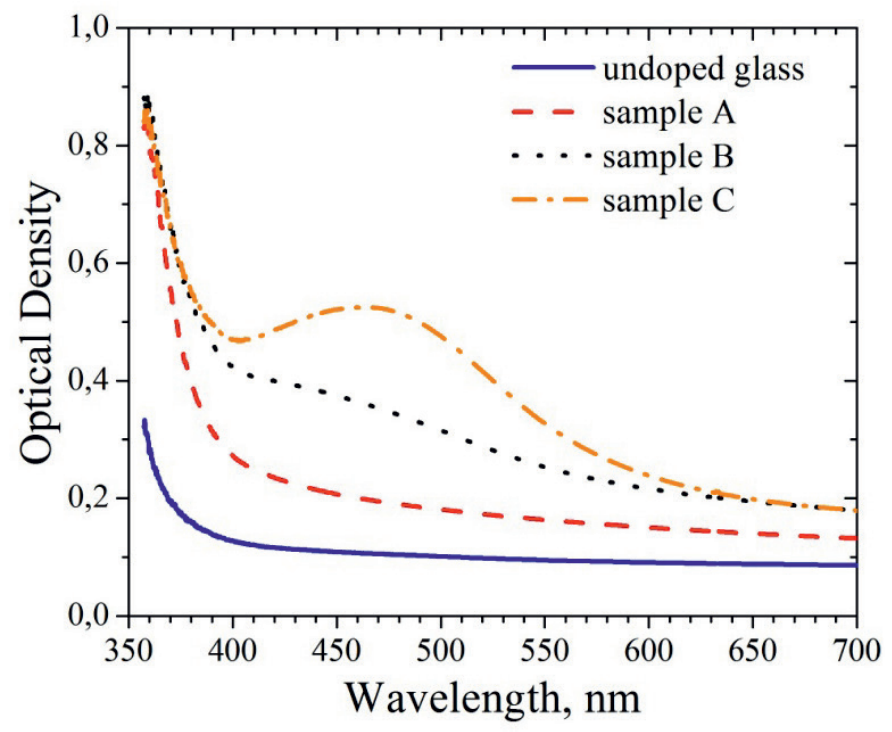

Figure 3. Optical density ( $O D=-\log _{10}(T)$ ) of the undoped glass (blue curve), glass containing amorphous $1.2 \mathrm{~nm}$ Ag nanoclusters (red curve), amorphous $2.4 \mathrm{~nm}$ Ag nanoparticles (black curve) and $2.8 \mathrm{~nm}$ Ag nanoparticles (orange curve).

\section{Nonlinear absorption measured by z-scan experiments}

The normalized transmittance of the samples, as measured in an open-aperture z-scan experiment, is shown in Fig. 4. When the sample is positioned far from the focal plane, the incident laser irradiance is low and transmittance is constant. Around the focal plane optical nonlinearity affects the absorption coefficient. All z-scan curves show a valley around $z=0$, corresponding to a decrease of the transmittance near the focal plane, i.e., optical limiting. The zscan curves measured on sample C (Fig. 4c) and to a lesser extent on sample B (Fig. 4b) show, in addition, two humps flanking the valley, which indicates that absorption saturation takes place. 
The overall shape of the transmittance curves measured for both Ag nanoclusters and Ag nanoparticles dispersed in glass are in a good agreement with previous experimental results obtained in solution, ${ }^{3,5,24}$ and for $\mathrm{Ag}: \mathrm{TiO}_{2}$ nanocomposites. ${ }^{25}$

To model the z-scan curves in Fig. 4 the nonlinear absorption coefficient $\alpha$ defined by: ${ }^{3,5}$

$$
\alpha(I)=\frac{\alpha_{0}}{1+I / I_{s}}+\beta I
$$

Herein, $\beta$ is the nonlinear absorption coefficient, $I_{s}$ is the absorption saturation, and $I$ is the incident irradiance. Eq. (1) reflects a simplified physical picture, which assumes that the electronic levels involved into absorption process are not degenerate. Further improvement of the model is only possible for well defined cases, where the electronic level structure is known. ${ }^{30}$ If $I$ $<<I_{s}$, the absorption does not saturate and $\alpha(I)=\alpha_{0}+\beta I$. For low incident irradiances, $\beta I$ can be neglected and the absorption does not depend on incident irradiance, corresponding to the linear regime. Along the axis of the Gaussian beam, $z$, and with $r$ the radial distance from the beam axis, the irradiance incident on the sample changes as:

$$
I(r, z)=I_{0}\left[\frac{\rho_{0}}{\rho(z)}\right]^{2} \exp \left[-\frac{2 r^{2}}{\rho^{2}(z)}\right] \text {. }
$$

where $I_{0}$ is the laser irradiance at the focal plane for $r, z=0$ and $\rho(z)$ is the radius of the laser beam, which depends on $z$ as $\rho(z)=\rho_{0} \sqrt{1+\left(z / z_{R}\right)^{2}}$ where $\rho_{0}$ is the beam radius in the beam waist and $z_{\mathrm{R}}$ is the Rayleigh range. Within the glass medium the irradiance changes, in addition to the focusing, because of the absorption:

$$
\frac{d I\left(z^{\prime}\right)}{d z^{\prime}}=-\alpha\left(I\left(z^{\prime}\right)\right) I\left(z^{\prime}\right)
$$

wherein $z^{\prime}$ is an internal coordinate in the sample, $z^{\prime} \in[0, L]$, parallel to the z-axis. Eq. (3) corresponds to the Beer-Lambert law if the optical medium is linear $\left(\alpha=\alpha_{0}\right)$. 
The irradiance upon leaving the sample, and thus the sample transmittance, can be obtained by solving Eq. (3) numerically for a given incident irradiance. Doing this for all $I(0, z)$ along the zaxis using Eq. (2) gives a transmittance curve as a function of two parameters $\beta$ and $I_{s}$. Fitting the simulated curve to the measured transmittance, normalized for the transmittance in the linear regime, allows deriving those parameters. Details of the fitting procedure are provided in the ESI. Although the fit is a function of two parameters, one can explicitly define the values of both because they affect the fitting curve differently. Whereas the absorption saturation $I_{s}$ is responsible for an increase of the transmittance over unity, the nonlinear absorption coefficient $\beta$ is responsible for the valley at high irradiance (in the center of the z-scan curve).
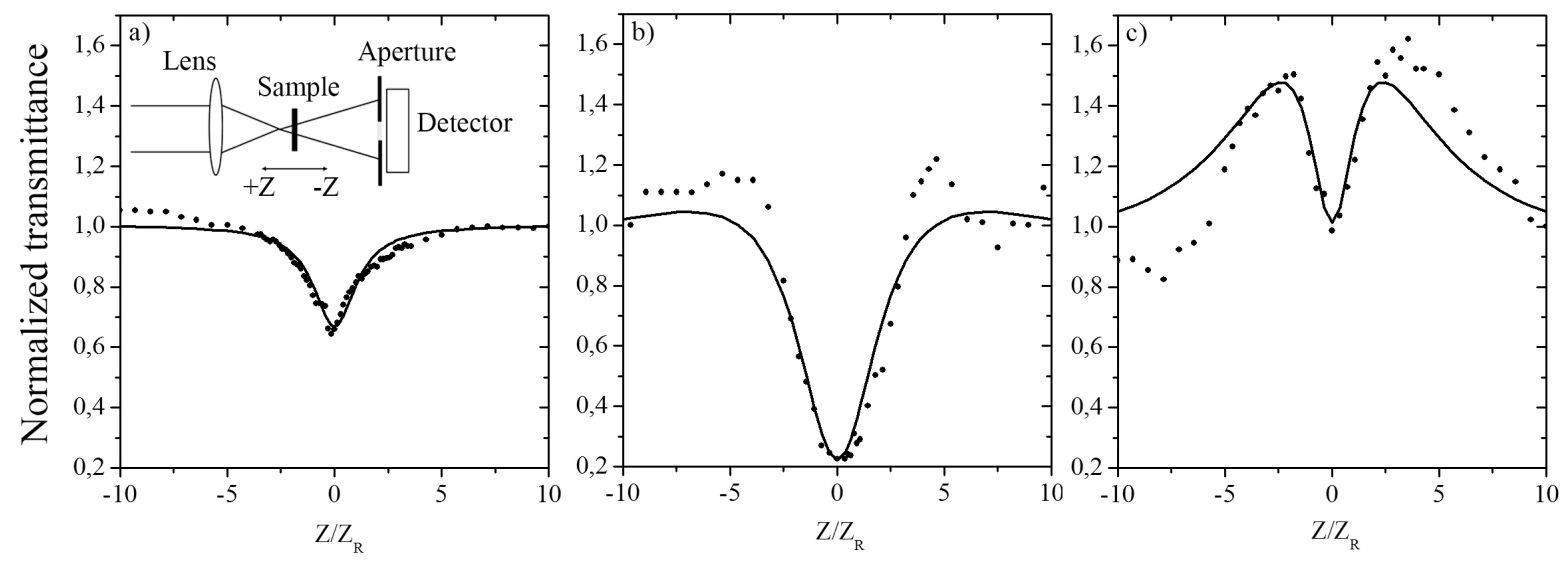

Figure 4.Picture will be updated for new alpha = minor change Open aperture z-scans for (a) sample A, (b) sample B, and (c) sample C. A scheme of used z-scan setup is shown as inset in (a). The evolution of nonlinear absorption behavior induced by heat treatment of the glass from nonsaturated absorption for nanoclusters (a) to saturated absorption for larger nanoparticles (b, c) is demonstrated. The solid lines are fits to the data using Eq. (3).

The fits are included in Fig. 4 and the corresponding values of $I_{s}$ and $\beta$ are summarized in Table 1. The uncertainty ranges are related with the quality of the fit and have been set to values corresponding to an increase of sum of squares of the residuals by $20 \%$.

The absorption coefficients $\alpha_{0}$ and $\beta$ can be converted into one- and two-photon absorption cross-sections $\sigma$ and $\delta$, respectively, if one knows the concentration of the particles, $D$ via 
$\sigma=\alpha / D$ and $\delta=\beta h v / D$ with $h v$ the photon energy. The order of magnitude of the particle concentration can be estimated from the known atomic percentage of $\mathrm{Ag}(0.15$ at.\%), the average Ag particle diameter, and the ratio of the volume of a Ag atom to an average atomic volume of the glass host. This gives concentrations of $(3-30) \times 10^{24} / \mathrm{m}^{3}$ for sample A, (5-40) $\times 10^{23} / \mathrm{m}^{3}$ for sample B, and (3-20) $\times 10^{23} / \mathrm{m}^{3}$ for sample C. The absorption cross sections $\sigma$ and $\delta$ are listed in Table 1.

Table 1. Linear $\left(\alpha_{0}\right)$ and nonlinear $(\beta)$ absorption coefficients, absorption saturation intensity $\left(I_{s}\right)$, and the one- $(\sigma)$ and two-photon $(\delta)$ absorption cross-sections of the studied Ag doped glass samples. (to check if molecular extinction coefficients are realistic)

\begin{tabular}{cccccc}
\hline $\begin{array}{c}\text { sample (average Ag } \\
\text { particle diameter) }\end{array}$ & $\alpha_{0}\left[10^{2} \mathrm{~m}^{-1}\right]$ & $\beta\left[10^{-10} \mathrm{~m} / \mathrm{W}\right]$ & $I_{s}\left[\mathrm{~W} / \mathrm{m}^{2}\right]$ & $\sigma\left[10^{-22} \mathrm{~m}^{2}\right]$ & $\delta\left[10^{6} \mathrm{GM}\right]$ \\
\hline sample A $(1.2 \mathrm{~nm})$ & $3.2 \pm 0.3$ & $4.5 \pm$ to do & $>10^{12}$ & $0.1-1.0$ & $0.04-0.8$ \\
& $5.8 \pm 0.5$ & $40 \pm$ to do & $\begin{array}{c}(0.3 \pm \text { to do }) \times \\
10^{10}\end{array}$ & $2.0-14$ & $3-40$ \\
sample B (2.4 nm) & & & & \\
& $8.9 \pm 0.8$ & $14 \pm$ to do & $\begin{array}{c}(10 \pm \text { to } \\
\text { do }) \times 10^{10}\end{array}$ & $4-35$ & $2-25$ \\
sample C (2.8 nm) & & & &
\end{tabular}

Uncertainty ranges are related to the uncertainty on the sample thickness for $\alpha_{0}$ and refer to statistical errors on the fit for $\beta$ and $I_{s}$. For $\sigma$ and $\delta$ the uncertainty ranges depend on the uncertainty on the particle concentration and to those on $\alpha_{0}$ and $\beta$, respectively

Glasses with small Ag clusters do not show absorption saturation under the current conditions $\left(I_{s}>10^{12} \mathrm{~W} / \mathrm{m}^{2}\right)$ and the nonlinear absorption coefficient of $\beta=4.5 \times 10^{-10} \mathrm{~m} / \mathrm{W}$ is comparable with the nonlinear absorption coefficient obtained for Au clusters dispersed in solution. ${ }^{3}$ The calculated two-photon absorption cross section $\delta$ of $0.04-0.8 \times 10^{6} \mathrm{GM}\left(1 \mathrm{GM}=10^{-58} \mathrm{~m}^{4} \mathrm{~s}\right.$ per molecule and per photon) is comparable to that obtained for monolayer protected $\mathrm{Au}_{25}$ clusters $0.427 \times 10^{6}$ GM. ${ }^{4,29}$ Upon annealing the atomic clusters agglomerate to form larger amorphous particles and eventually some larger polycrystalline particles appear (sample C only). This change results in a stronger light absorption at the SPR wavelength and absorption saturation at 
moderate irradiances (Figs. 4b and 4c). The largest $\beta$ and $\delta$ values are obtained for the sample containing $2.4 \mathrm{~nm}$ Ag nanoparticles (sample B). A similar maximum of the nonlinear absorption coefficient accompanied with lowering of the absorption saturation threshold was also observed with increase of the Au clusters' size in solution. In ref. [3] $\mathrm{Au}_{144}$ clusters, corresponding to a particles size of about $2 \mathrm{~nm}$ had the largest $\beta$. In ref. [29] a maximum in the two-photon absorption cross section of $1.476 \times 10^{6} \mathrm{GM}$ was found for $\mathrm{Au}_{309}$ in hexane, which is slightly lower than the values found in the current work for sample B (with particles of a comparable size). Further heat treatment of the glass sample leads to increase of the Ag nanoparticles size (sample C) and shows a decrease of the $\beta$ and $\delta$ value.
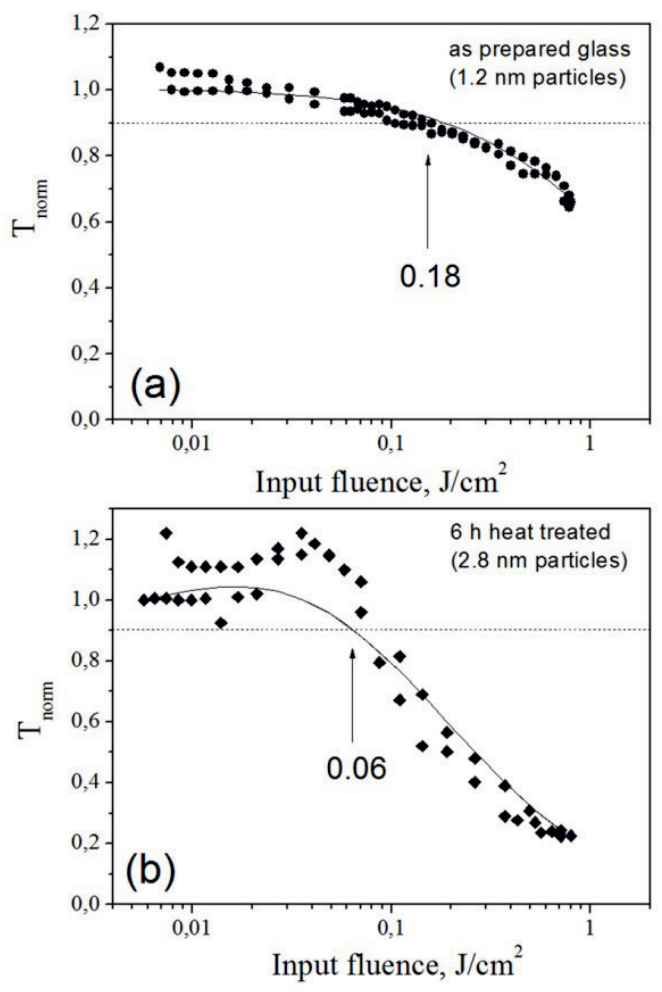

Figure 5. Figure still to be finetuned by Vladimir (layout + change threshold to 75\%) Normalized transmission of sample A (a) and sample B (b) versus incident laser fluence. The decrease of the optical limiting threshold induced by heat treatment of the glass is demonstrated. The solid lines are fits to the data using Eq. (3). 
The normalized transmittance of samples A and B is plotted as a function of the input fluence (Fig. 5). The transmittance remains almost the same at low input fluences and decreases beyond some input fluence level. Defining the optical limiting thresholds, $F_{t}$, as the fluence at which the transmission drops to $75 \%$ of the linear transmission, thresholds of $0.4 \mathrm{~J} / \mathrm{cm}^{2}$ and $0.15 \mathrm{~J} / \mathrm{cm}^{2}$ are found for samples A and B, respectively. It is to be noted that the optical limiting threshold gets lowered as the Ag particles become larger. Moreover, the optical limiting threshold found here are lower than those of other materials with good optical limiting properties, such as $\mathrm{Ag}: \mathrm{TiO}_{2}$ nanocomposites [25] and ligand protected gold clusters in toluene [3], demonstrating the viability of the samples as ideal optical limiting materials.

\section{Discussion}

Sample A contains Ag clusters with a size of $(1.2 \pm 0.4) \mathrm{nm}$, which assuming a spherical cluster geometry and a bulk density corresponds to $15-125$ atoms with a most probable size of $\sim 50$ atoms. Experimental studies of silver clusters in an argon matrix demonstrate that the optical absorption spectra of clusters containing less than about 15 atoms demonstrate molecular-like absorptions in the $3.0-5.0 \mathrm{eV}$ with strong size-to-size variations. ${ }^{27,28}$ For larger clusters the spectra evolve from molecular-like to a plasmon-like with increasing size. Calculated optical spectra show the appearance of plasmon-like features in the size range of 20 to 120 atoms. ${ }^{20}$ The spectra have one intense absorption band, which position varies linearly in a region of 3.6-3.0 eV with the inverse size of the Ag clusters (1.04-2.21 nm for 20-120 atoms), and a less intense tail in the higher energy range $(4.0-5.5 \mathrm{eV}){ }^{9,20,27}$ The main optical transitions in the absorption spectrum originate from $\mathrm{sp} \rightarrow \mathrm{sp}$ intraband transition, corresponding to the plasmon resonance for larger particles, while less intense $\mathrm{d} \rightarrow$ sp interband transitions contribute at higher energies. ${ }^{20}$ For 
$\operatorname{Ag}_{n}(n=10-22)$ clusters, molecular orbitals near the Fermi level, $E_{f}$, have mostly sp character because the d-band is situated more than $3 \mathrm{eV}$ below $E_{f .}{ }^{21}$

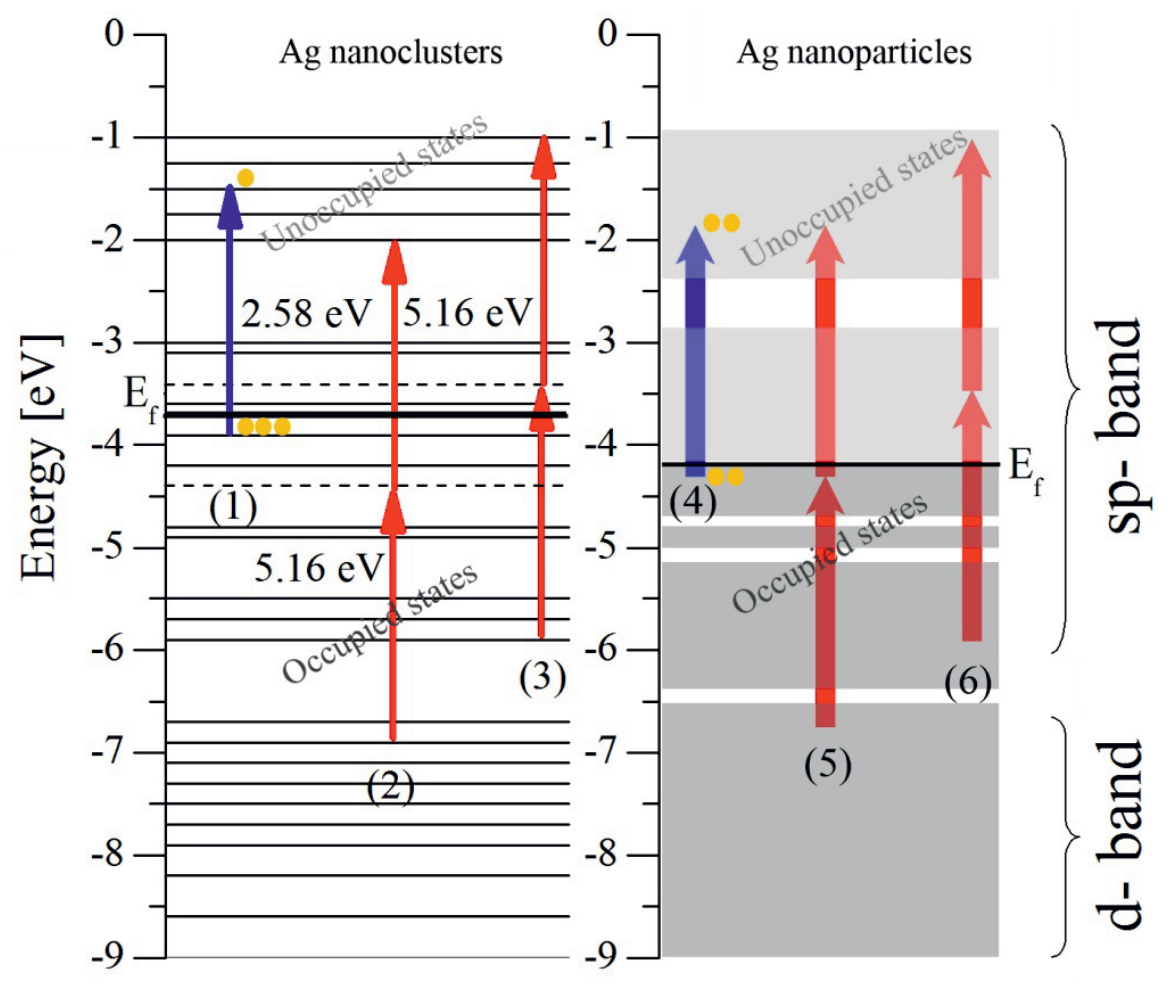

Figure 6. Schematic energy level diagram of electronic excitation transitions using 2.58 $\mathrm{eV}$ photons in Ag nanoclusters (a) and nanoparticles (b). Single photon optical absorption in clusters and collective excitation near the plasmon resonance for particles are colored blue. Two-photon absorptions via virtual states are colored red. Not equal and equal population electron population of upper and lower electronic states corresponding to linear absorption for clusters (a) and absorption saturation for nanoparticles (b) are presented by yellow circles.

Since the glass matrix that surrounds the Ag nanoclusters is assumed to change the energy spectra only quantitatively, this theoretical information can be used to explain the optical density spectrum of sample A in figure 3. The intense absorption below $400 \mathrm{~nm}$ originates mainly from $\mathrm{sp} \rightarrow \mathrm{sp}$ transitions, and the less intense absorption tail, stretching from 400 to $700 \mathrm{~nm}$ may be related to the size distribution (and the shift of the absorption to the red with increasing cluster 
size). A hypothetic energy level diagram is proposed to explain the observed nonlinear absorption measured by the z-scan experiments (Fig. 6a). At low incident beam irradiance only a single photon molecular-like absorption takes place at under the used excitation $(2.58 \mathrm{eV})$. Fast photoluminescent decay (between 5 and 100 ns depending on used preparation conditions) takes place in the glasses. ${ }^{31}$ Thus, depopulation of the upper level happens on a timescale comparable to population of the excited state. Meanwhile the absorption, opposed to the situation with the plasmonic particles, has non-resonant behavior and the population of the upper level. The $\mathrm{sp} \rightarrow \mathrm{sp}$ transition of the atomic clusters is thus not saturated.

Upon enhancement of the laser irradiance by moving the sample towards the beam waist twophoton absorption processes also become possible. These transitions can be both $\mathrm{d} \rightarrow \mathrm{sp}$ transitions (2 in Fig. 6a) from lower lying d-like orbitals and $s p \rightarrow$ sp excitations (3 in Fig. 6a) from levels below $E_{f}$ to unoccupied electronic states. Opening of the two-photon absorption channel, associated with $\beta$, leads to a decrease of the sample transmittance. This is observed as a central valley in the z-scan curve (Figs. 4a).

For the silver nanoparticles of samples C, the used excitation wavelength of $480 \mathrm{~nm}(2.58 \mathrm{eV})$ coincides with the SPR (4 in Fig. 6b). At low incident beam irradiance, corresponding to the case that the sample is far from the focus, the amount of transitions scales with the irradiance (linear regime). Excitation at the SPR corresponds to a high photon absorption probability. This leads to absorption saturation at moderate irradiance $\left(I(z) \sim 1 \times 10^{10} \mathrm{~W} / \mathrm{m}^{2}\right)$ due to electron population equalization of the lower unoccupied and upper occupied levels. The absorption saturation already takes place at the beginning of the investigated z-scan range and renders the sample more transparent. This is the origin of the humps in the transmittance on both sides of the central valley (Fig. 4c). Upon further enhancement of the laser irradiance two-photon absorption 
processes can take place, corresponding to $d \rightarrow$ sp interband transitions (5 in Fig. 6b) from lower lying d-like orbitals and $\mathrm{sp} \rightarrow \mathrm{sp}$ intraband excitations (6 in Fig. 6b) from levels below $E_{f}$. As is also the case for sample A, the opening of the two-photon absorption channel leads to a decrease of the sample transmittance that is observed as a central valley in the z-scan curve (Figs. 4c).

Sample B can be seen as a situation that is intermediate between sample A and sample B. The SPR is not completely developed (as seen in Figure 3), which implies a higher saturation intensity than in sample C. Nonetheless some absorption saturation is observed in Fig 4b, in combination with a reduced transmittance at high irradiance.

In agreement with the present results on the small amorphous Ag particles (samples A and B), the two-photon absorption cross section of Au clusters was also found to increase with cluster size. ${ }^{29}$ It should be noted that for Au clusters the d-band is situated closer to the sp-band and the one photon $\mathrm{d} \rightarrow \mathrm{sp}$ interband transitions can occur for the $2.58 \mathrm{eV}$ excitation used, while for $\mathrm{Ag}$ clusters the energy of a single photon is not sufficient to excite $\mathrm{d} \rightarrow$ sp transitions. The reduced non-linear absorption coefficient for sample C, as compared to sample B, is likely related to further increase of the particles size. In addition the formation of large polycrystalline nanoparticles may have an effect on the non-linear absorption.

\section{Conclusion}

Optical nonlinear absorption of Ag nanoclusters ( $1.2 \mathrm{~nm}$ diameter) and Ag nanoparticles ( $2.8 \mathrm{~nm}$ diameter) dispersed within a transparent oxyfluoride glass host was studied using a $\mathrm{z}$ scan experiment following excitation close to the plasmon resonance of the nanoparticles.

Upon annealing of the samples, the Ag particles become larger, which is reflected in an evolution of nonlinear absorption properties from a non-saturated to the saturable regime under 
incident laser irradiances of $10^{10}-10^{12} \mathrm{~W} / \mathrm{m}^{2}$. With increase of the Ag particle size from $1.2 \mathrm{~nm}$ to $2.4 \mathrm{~nm}$, the non-linear absorption coefficient of the glasses is enhanced from $4.5 \times 10^{-10}$ to $40 \times 10^{-10} \mathrm{~m} / \mathrm{W}$, associated with absorption saturation appearance $I_{s} \approx 10^{10} \mathrm{~W} / \mathrm{m}^{2}$. Further annealing of the samples leads to the formation of larger amorphous and a few polycrystalline nanoparticles that show a clear SPR band, absorption saturation, and a reduction of the nonlinear absorption coefficient to $14 \times 10^{-10} \mathrm{~m} / \mathrm{W}$. In addition, a reduction of the optical limiting threshold by moderate heat treatment of the samples is found.

Absorption saturation in nanoparticles even at low irradiances originates from the electron population equalization at the lower and upper levels of the occupied and unoccupied states via resonant $\mathrm{sp} \rightarrow \mathrm{sp}$ intraband transitions. Two-photon absorption channels open at higher irradiance due to the $\mathrm{d} \rightarrow \mathrm{sp}$ interband and $\mathrm{sp} \rightarrow \mathrm{sp}$ intraband excitations. Opposite to plasmonic particles, the $\mathrm{sp} \rightarrow \mathrm{sp}$ intraband absorption is not saturated for atomic clusters and the opening of two-photon absorption channels has only an additive character. The non-linear absorption properties of these glasses doped with small Ag nanoclusters are promising for applications in optical limiting and object's contrast enhancement.

\section{Acknowledgments}

This work is supported by the Research Foundation - Flanders (FWO) and by the KU Leuven Research Council (BOF, GOA, and IDO programs). The authors are grateful to Methusalem funding by the Flemish government. M.V.S. acknowledges to the FWO for a PhD fellowship. S.T. acknowledges the FWO for a post-doctoral fellowship. Part of this work was supported by 
funding from the European Research Council under the 7th Framework Program (FP7), ERC grant $\mathrm{N}^{\circ} 246791$ - COUNTATOMS and the EC project IFOX.

\section{REFERENCES}

1. Palpant, B. "Third-Order Nonlinear Optical Response of Metal Nanoparticles" in NonLinear Optical Properties of Matter, Papadopoulos, M.G. et al., eds.; Springer (Dordrecht, The Netherlands), 2006, pp. 461-508.

2. Banfi, G.; Degiorgio, V.; Ricard, P. Nonlinear Optical Properties of Semiconductor Nanocrystals. Adv. Phys. 1998, 47, 447-510.

3. Philip, R.; Chantharasupawong, P.; Qian, H.; Jin, R.; Thomas, J. Evolution of Nonlinear Optical Properties: From Gold Atomic Clusters to Plasmonic Nanocrystals. Nano Lett. 2012, 12, 4661-4667.

4. Yau, S. H.; Varnavski, O.; Goodson, T. An Ultrafast Look at Au Nanoclusters. Accounts Chem. Res. 2013, 45, 1506-1516.

5. Hari, M.; Mathew, S.; Nithyaja, B.; Joseph, S. A.; Nampoori, V. P. N.; Radhakrishnan, P. Saturable and Reverse Saturable Absorption in Aqueous Silver Nanoparticles at OffResonant Wavelength. Opt. Quant. Electron. 2012, 43, 49-58.

6. Tikhomirov, V. K.; Rodríguez, V. D.; Kuznetsov, A. S.; Kirilenko, D.; Van Tendeloo, G.; Moshchalkov, V. V. Preparation and Luminescence of Bulk Oxyfluoride Glasses Doped with Ag Nanoclusters. Opt. Express 2010, 18, 22032-22040. 
7. Tikhomirov, V.K.; Vosch, T.; Fron, E.; Rodríguez, V.D.; Velázquez, J.J.; Kirilenko, D.; Van Tendeloo, G.; Hofkens, J.; Van der Auweraer, M.; Moshchalkov, V. V. Luminescence of Oxyfluoride Glasses co-Doped with Ag Nanoclusters and $\mathrm{Yb}^{3+}$ Ions. RSC Adv. 2012, 2, $1496-1501$.

8. Karthikeyan, B.; Tholas, J.; Philip, R. Optical Nonlinearity in Glass-Embedded Silver Nanoclusters under Ultrafast Laser Excitation. Chem. Phys. Lett. 2005, 414, 346-350.

9. Baishya, K.; Idrobo, J. C.; Öğüt, S.; Yang, M.; Jackson, K.; Jellinek, J. Optical Absorption Spectra of Intermediate-Size Silver Clusters from First Principles. Phys. Rev. B. 2008, 78, 075439 (9pp).

10. Idrobo, J. C.; Walkosz, W.; Yip, S. F.; Ögüt, S.; Wang, J.; Jellinek, J. Static Polarizabilities and Optical Absorption Spectra of Gold Clusters $\left(\mathrm{Au}_{n}, n=2-14\right.$ and 20) from first principles. Phys. Rev. B. 2007, 76, 205422.

11. Fedrigo, S.; Harbich, W.; Buttet, J. Collective Dipole Oscillations in Small Silver Clusters Embedded in Rare-Gas Matrices. Phys Rev. B. 1993, 47, 10706-10715.

12. Coutino-Gonzalez, E.; Roeffaers, M. B. J.; Dieu, B.; De Cremer, G.; Leyre, S.; Hanselaer, P.; Fyen, W.; Sels, B.; Hofkens, J. Determination and Optimization of the Luminescence External Quantum Efficiency of Silver-Clusters Zeolite Composites. J. Phys. Chem. C 2013, 117, 6998-7004.

13. Sharma J.; Yeh H. C.; Yoo H.; Werner J. H.; Martinez J. S. A Complementary Palette of Fluorescent Silver Nanoclusters. Chem. Commun. 2010, 46, 3280-3282. 
14. Zheng J.; Petty J. T.; Dickson R. M. High Quantum Yield Blue Emission from WaterSoluble $\mathrm{Au}_{8}$ Nanodots. J. Am. Chem. Soc. 2003, 125, 7780-7781.

15. Tikhomirov, V. K.; Furniss, D.; Reaney, I. M.; Beggiora, M.; Ferrari, M.; Montagna, M.; Rolli, R. Fabrication and Characterization of Nanoscale, $\mathrm{Er}^{3+}$-Doped, Ultratransparent Oxy-fluoride Glass Ceramics. Appl. Phys. Lett. 2002, 81, 1937-1939.

16. Bahae, M. S.; Said, A. A.; Van Stryland, E. W. High-Sensitivity, Single Beam $\mathrm{n}_{2}$ Measurements. Opt. Lett. 1989, 14, 955-957.

17. Bahae, M. S.; Said, A. A.; Wei, T. H.; Hagan, D. J.; Van Stryland, E. W. Sensitive Measurement of Optical Nonlinearities Using a Single Beam. IEEE J. Quantum Electron. 1990, 26, 760-769.

18. Langhammer, C.; Kasemo, B.; Zorić, I. Absorption and Scattering of Light by Pt, Pd, Ag, and Au Nanodisks: Absolute Cross Sections and Branching Ratios. J. Chem. Phys. 2007, 126, 194702 (11pp).

19. Shestakov, M. V.; Meledina, M.; Turner, S.; Tikhomirov, V. K.; Verellen, N.; Rodríguez, V. D.; Velázquez, J. J.; Van Tendeloo, G.; Moshchalkov, V. V. The Size and Structure of Ag Particles Responsible for Surface Plasmon Effects and Luminescence in Ag Homogeneously Doped Bulk Glass. J. Appl. Phys. 2013, 114, 073102 (5pp).

20. Aikens, C. A.; Li, S.; Schatz, G. C. From Discrete Electronic States to Plasmons: TDDFT Optical Absorption Properties of $\operatorname{Ag}_{n}(n=10,20,35,56,84,120)$ Tetrahedral Clusters. $J$. Phys. Chem. C. 2008, 112, 11272-11279. 
21. Pereiro, M.; Baldomir, D. Structure of Small Silver Clusters and Static Response to an External Electric Field. Phys. Rev. A. 2007, 75, 033202 (10pp).

22. Kassab, L. R. P., de Almeida, R. ; da Silva, D. M.; de Assumpção, T.A.A.; de Araújo, C.B. ; Enhanced Luminescence of $\mathrm{Tb}^{3+/} \mathrm{Eu}^{3+}$ Doped Tellurium Oxide Glass Containing Silver Nanostructures. J. Appl. Phys. 2009, 105, 103505 (3pp).

23. de Assumpção, T. A. A.; da Silva, D. M.; Camilo, M. E.; Kassab, L. R. P.; Gomes, A. S. L.; de Araújo, C. B.; Wetter, N. U. Frequency Upconversion Properties of $\mathrm{Tm}^{3+}$ Doped $\mathrm{TeO}_{2}-\mathrm{ZnO}$ Glasses Containing Silver Nanoparticles, J. Alloy. Compd. 2012, 536, S504-S506.

24. Kumar, S.; Shibu, E. S.; Pradeep, T.; Sood, A. K. Ultrafast Photoinduced Enhancement of Nonlinear Optical Response in 15-atom Gold Clusters on Indium Tin Oxide Conducting Film. Opt. Express 2013, 21, 8483-8492.

25. Hari, M.; Joseph, S. A.; Mathew, S.; Radhakrishnan, P.; Nampoori, V. P. N. Band-Gap Tuning and Nonlinear Optical Characterization of Ag: $\mathrm{TiO}_{2}$ Nanocomposites. J. Appl. Phys. 2012, 112, 074307 (8pp).

26. Gao, Y.; Zhang, X. R.; Li, Y. L.; Liu, H. F.; Wang, Y. X.; Chang, Q.; Jiao, W. Y.; Song, Y. L. Saturable Absorption and Reverse Saturable Absorption in Platinum Nanoparticles. Opt. Commun. 2005, 251, 429-433.

27. Fedrigo, S.; Harbich, W.; Belyaev, J.; Buttet, J.; Evidence for Electronic Shell Structure of Small Silver Clusters in the Optical Absorption Spectra. Chem. Phys. Lett. 1993, 211, 166170. 
28. Harb, M.; Rabilloud, F.; Simon, D.; Rydlo, A.; Lecoultre, S.; Conus, F.; Rodrigues, V. and Félix, C. Optical Absorption of Small Silver Clusters: Ag, $(n=4-22)$. J. Chem. Phys. 2008, 129, 194108 (9pp).

29. Ramakrishna, G.; Varnavski, O.; Kim, J.; Lee, D.; Goodson, T.; Quantum-Sized Gold Clusters as Efficient Two-Photon Absorbers. J. Am. Chem. Soc. 2008, 130, 5032-5033.

30. Sagle, J.; Namiotka, R. K.; Huennekens, J. Measurement and modelling of intensity dependent absorption and transit relaxation on the cesium D1 line, J. Phys. B: Mol. Opt. Phys. 1996, 29, 2629-2643

31. Kuznetsov, A. S.; Velázquez, J. J.; Tikhomirov, V. K.; Mendez-Ramos, J.; Moshchalkov, V. V. Quantum yield of luminescence of Ag nanoclusters dispersed within transparent bulk glass vs. glass composition and temperature. Appl. Phys. Lett. 2012, 101, 251106 (5pp). 